\title{
Effects of ingestion of organic selenium in adapted and non-adapted rats
}

\author{
BY W. G. JAFFE AND CRISTINA MONDRAGON \\ Instituto Nacional de Nutrición, Apartado 2049, Caracas, Venezuela
}

(Received I6 May 1974-Accepted 17 Fune 1974)

\begin{abstract}
I. Rats were given moderate-selenium $(4.5 \mathrm{mg} / \mathrm{kg})$ or low-Se $(0.5 \mathrm{mg} / \mathrm{kg})$ diets during gestation and lactation. Their young were given diets with high $(10 \mathrm{mg} / \mathrm{kg})$, moderate or low Se contents from weaning, and groups of rats were killed at intervals during the 14 -week experimental period.

2. Compared with young rats which received the low-Se diet, those which received the moderate- or high-Se diets had a high incidence of liver lesions and there were changes in liver Se content, haemoglobin concentration, packed cell volume, prothrombin activity, fibrinogen content, spleen weight, body water and serum glutamic-oxaloacetic and glutamic-pyruvic transaminase (L-aspartate:2-oxoglutarate aminotransferase; $E C$ 2.6.1. I and L-alanine: 2-oxoglutarate aminotransferase; $E C$ 2.6.1.2 respectively) and alkaline phosphatase ( $E C_{3}$ 3.I.3.I) activities. In those rats which received the high-Se diet the changes were more pronounced than in those which received the moderate-Se diet.

3. In young rats from dams given moderate-Se diets, which were themselves given the moderate-Se diet, the liver Se content decreased continuously, whereas rats given the same diet but from dams which had received the low-Se diet, the liver Se content increased continuously. There was a slight improvement of symptoms of Se toxicity in all groups by the 5 th week of the experimental period.
\end{abstract}

4. The results suggest that there was an adaptation to chronic Se intake.

The study of the prolonged action of selenium is of special interest in view of the fact that animals and humans living in seleniferous areas may be exposed to the effects of Se throughout their lives and for generations. Most of the long-term studies of Se toxicity have involved the use of inorganic salts of Se, although there are wellrecognized differences between their effects and those due to organic forms of the element, e.g. those found in food products and animal foods (Smith \& Lillie, 1940).

Although there are numerous publications about the toxic effects of Se in animals (Rosenfeld \& Beath, 1964), our knowledge of the physiological and pathological changes which occur in chronic selenosis is still incomplete.

In previous experiments, we found indications of an adaptation to moderate doses of Se (Jaffé \& Mondragón, 1969). We have also reported (Jaffé, Mondragón, Layrisse \& Ojeda, 1972), that the administration of organic Se to rats over a period of 6 weeks produced changes in the activities of some serum enzymes as well as in other blood factors. We suggested that the variations in these activities might be useful for the diagnosis of human selenosis.

In the present paper we report on the changes in activity of serum enzymes and some other indices of selenosis in young rats from dams given moderate-Se or low-Se diets. 
Table 1 . Composition $(\mathrm{g} / \mathrm{kg})$ of the experimental diets given to rats

\begin{tabular}{|c|c|c|c|}
\hline & & Diets & \\
\hline & Moderate-Se & Low-Se & High-Se \\
\hline Selenium-containing sesame-seed meal & $230 \cdot 8$ & - & $490 \cdot 2$ \\
\hline Se-free sesame-seed meal & 270.4 & $463 \cdot 3$ & $\longrightarrow$ \\
\hline $\mathrm{L}-\mathrm{l}$ ysine $\mathrm{HCl}$ & $4 \cdot 0$ & $4 \cdot 0$ & $4^{\circ} \cdot$ \\
\hline Mineral salts* & $40 \cdot 0$ & $40 \cdot 0$ & $40 \cdot 0$ \\
\hline Maize oil & $50 \cdot 0$ & $50 \cdot 0$ & $50^{\circ} \mathrm{o}$ \\
\hline Cod-liver oil & 10.0 & 10.0 & $10 \cdot 0$ \\
\hline Vitamin mixturef & $10 \cdot 0$ & $10 \cdot 0$ & $10 \cdot 0$ \\
\hline Starch & $384 \cdot 8$ & $422 \cdot 7$ & $395 \cdot 8$ \\
\hline Se content $(\mathrm{mg} / \mathrm{kg})$ & $4 \cdot 5$ & 0.5 & IO \\
\hline
\end{tabular}

* US Pharmacopeia, XIV (I960); with (g/kg mixture): $0 \cdot 10$ copper, $1 \cdot 25$ manganese and $0 \cdot 30$ zinc.

$\uparrow$ To give $(\mathrm{mg} / \mathrm{kg}$ diet): thiamin 3, riboflavin 3, pyridoxine 2, calcium pantothenate 2o, nicotinic acid 20 , pteroylmonoglutamic acid 0.25 , biotin 0.10 , cyanocobalamin 0.015, myo-inositol 100, choline I000.

\section{EXPERIMENTAL}

\section{Diets}

The composition of the diets used is given in Table 1 . The Se contents of the lowSe, moderate-Se and high-Se diets were $0.5,4.5$ and $10.0 \mathrm{mg} / \mathrm{kg}$ respectively.

\section{Experimental design}

Female Sprague-Dawley albino rats were given a low-Se or moderate-Se diet ad lib. during gestation and lactation. The offspring of these rats remained with their dams until weaning at $2 \mathrm{I} d$ of age and were then removed and given one of the three diets.

Group A ("non-adapted' rats) consisted of seventy-seven rats from dams which had received the low-Se diet and were divided into two subgroups: A-I, thirty-nine rats which were given the moderate-Se diet and A-2, thirty-two rats which were given the high-Se diet.

Group B ('adapted' rats) consisted of seventy-four rats from dams which had received the moderate-Se diet and were divided into two subgroups: B-I, thirty-five rats which were given the moderate-Se diet and B-2, thirty-three rats which were given the high-Se diet.

Group C (control rats) consisted of thirty-three rats from dams which had received the low-Se diet and remained on the same diet after weaning.

Liver Se levels only were determined at $24 \mathrm{~d}$ of age for five additional groups, each of six rats, which were treated identically to each of the five experimental groups.

\section{Methods of analysis}

Rats from each of the five experimental groups were killed on the $5^{\text {th, }} 7^{\text {th, }}$, oth or I 4 th week after weaning. For zero values, groups of six $2 \mathrm{I}$-d-old rats from groups A and $\mathrm{B}$ were used. The animals were weighed and blood samples were taken by cardiac puncture. The samples were mixed with $0^{\circ} \mathrm{I}$ M-sodium oxalate $(9: \mathrm{I}, \mathrm{w} / \mathrm{v})$. Plasma was assayed for prothrombin activity by the method of Quick (1957), for fibrinogen content by the Biuret method (Natelson, I96r $a$ ), for glutamic-oxaloacetic 
and glutamic-pyruvic (L-aspartate: 2-oxoglutarate aminotransferase; EC 2.6. I . I and L-alanine:2-oxoglatarate aminotransferase; $E C$ 2.6. I.2 respectively) trans activities by the method of Reitman \& Frankel (1957), using the DADE kit (DADE Division, American Hospital Supply Corporation, Miami, Florida 33 52 , USA) and for alkaline phosphatase (EC 3. I . 3.x) activity (Ohmori, I 937; King \& Delory, r 939 as modified by the Sigma Chemical Company, I963). Each blood sample was assayed for haemoglobin (Hainline, 1958) and packed cell volume (Natelson, I96I $b$ ). Liver Se was determined by the fluorometric method of Cummings, Martin \& Maag (1965) as modified by Jaffé, Chávez \& Mondragón (1967).

\section{Post-mortem examination}

After the withdrawal of blood, each animal was examined for the presence of lesions which may have resulted from Se intoxication. The spleen was removed and weighed. The liver was removed for the determination of Se content. Body-water content was calculated from the weights of the dried carcasses.

\section{RESULTS}

The lesions found on post-mortem examination included: speckled liver with pale yellow patches, irregularity of the lobular margins, nodular surface and reduction in liver size; hyperplastic spleen; oedema; inflammation of the pancreas; the presence of ascites. Fig. I $a$ shows that the animals of subgroups A-r ('non-adapted') and B-I ('adapted') had no macroscopic hepatic lesions during the first 7 weeks on the moderate-Se $(4.5 \mathrm{mg} \mathrm{Se} / \mathrm{kg})$ diet. After ro weeks on this diet the number of animals from the 'non-adapted' group A-I which were found to have liver lesions was significantly higher than that of the control group in which there were no animals with lesions. The difference between the number of rats from 'adapted' group B-I and the controls which were found to have liver lesions was not significant. With the diet containing Io $\mathrm{mg} \mathrm{Se} / \mathrm{kg}$ (high-Se) a high percentage of hepatic lesions was observed in animals of both subgroups $\mathrm{A}-2$ and $\mathrm{B}-2$ from the 5 th week on. The number of lesions in the 'adapted' rats was always lower than in the 'non-adapted' rats, but the difference was statistically significant only at 5 weeks (Table 2).

Mortality occurred only in the group of 'non-adapted' animals: $13.5 \%$ between 6 and 9 weeks after weaning and $8.3 \%$ between 9 and 12 weeks, with no further occurrence of spontaneous deaths. Animals which died were extremely pale, had anaemia, marked weight loss, liver cirrhosis, and hyperplasic spleens. The animals killed at each time of sampling had no apparent signs of illness.

Oedema and inflammation of the pancreas was found in $25 \%$ of the 'non-adapted' rats, 5 weeks after the beginning of the experiment and in $48 \%$ of these rats after 7 weeks on the high-Se diet.

A comparison of Fig. $\mathrm{I} a$ and $b$ shows that the pattern of liver Se was similar to that for the frequency of occurrence of hepatic lesions. 'Non-adapted' rats given the highSe diet accumulated the element rapidly up to the $5^{\text {th }}$ week after which time there was a gradual decrease in Se content of the liver. At weaning the 'adapted' rats had a high 

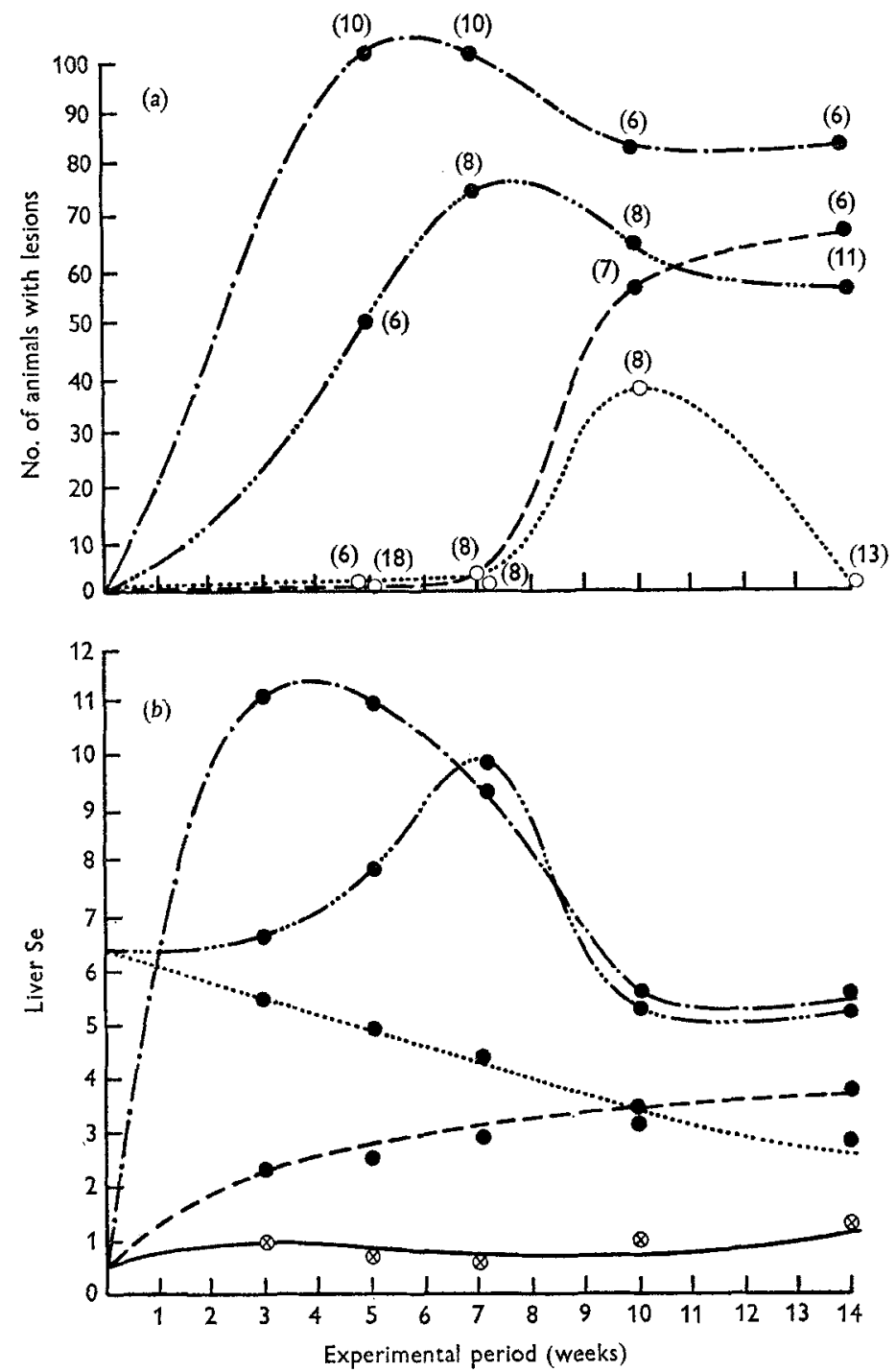

Fig. r. Effects of dietary selenium on the livers of rats. (a) No. of rats with macroscopic lesions $(\%)$; $(b)$ liver Se levels (mg/kg wet weight). - - ., A-r, 'non-adapted' rats (from dams given a low-Se diet; $0.5 \mathrm{mg} / \mathrm{kg}$ ) given a moderate-Se $\operatorname{diet}(4.5 \mathrm{mg} / \mathrm{kg})$ from weaning; - . - . A-2, 'non-adapted' rats given a high-Se diet from weaning; ...., B-r, 'adapted' rats (from dams given a moderate-Se diet) given a moderate-Se diet from weaning; $\cdots-\cdots, \mathbf{B}-2$, 'adapted' rats given a high-Se diet from weaning; - control rats (from dams given a low-Se diet) given a low-Se diet from weaning; $O$, values not significantly different from control values; 9 , values significantly different from control values: $P \leqslant 0.05 ; \otimes$, controls. None of the animals in the control group were found to have lesions, therefore this group is not represented in $(a)$. No. of animals killed at 5 th, 7 th, Ioth and 14 th weeks after weaning is given in parentheses. 

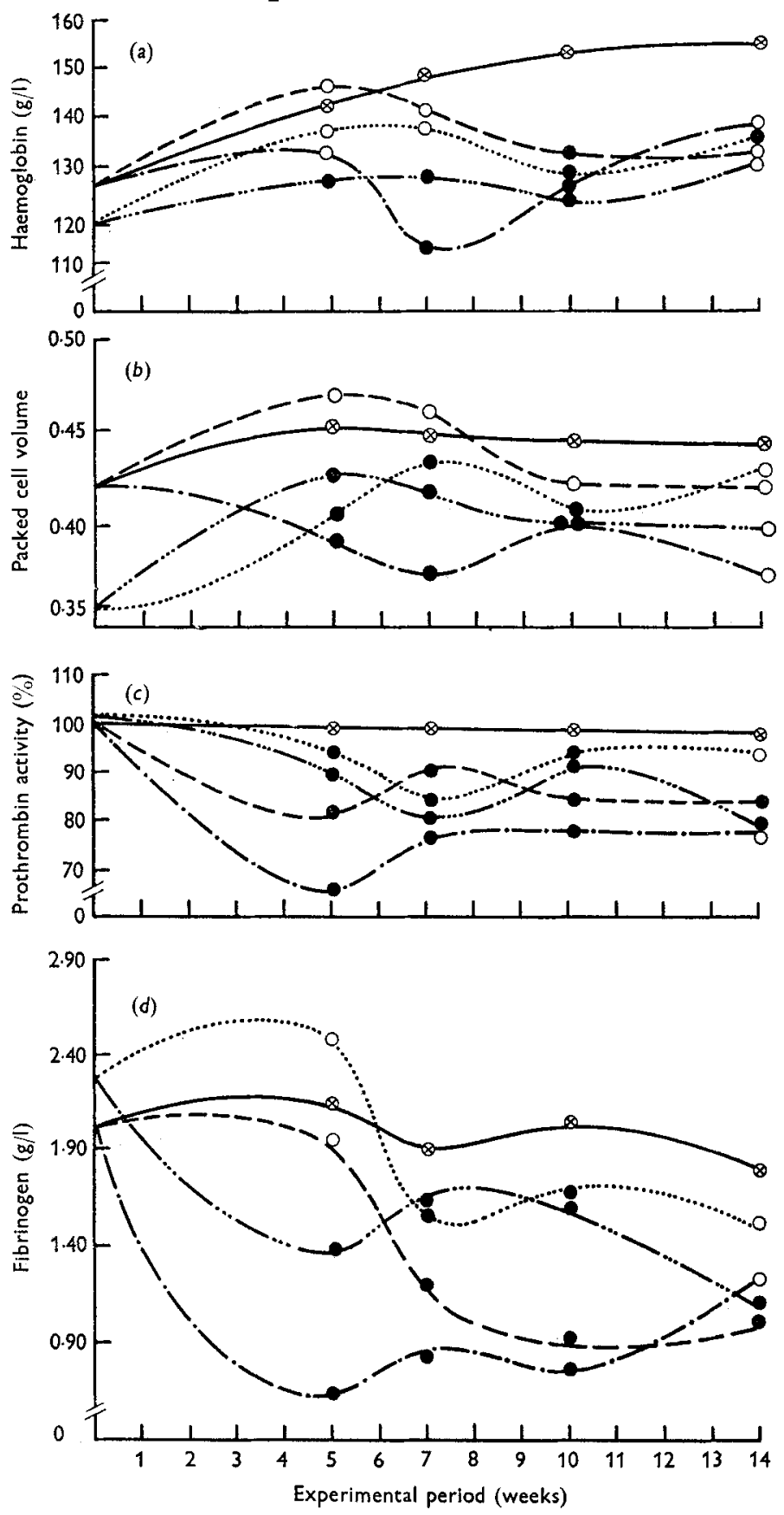

Fig. 2. Effects of dietary selenium on the blood of rats. (a) Haemoglobin levels, (b) blood packed cell volume, $(c)$ prothrombin activity, $(d)$ serum fibrinogen levels.,$---- A-1$, 'nonadapted' rats (from dams given a low-Se diet; $0.5 \mathrm{mg} / \mathrm{kg}$ ) given a moderate-Se diet $(4.5 \mathrm{mg} / \mathrm{kg}$ ) from weaning; -.-.-, A-2, 'non-adapted' rats given a high-Se diet ( $10 \mathrm{mg} / \mathrm{kg}$ ) from weaning; ....., B-I, 'adapted' rats (from dams given a moderate-Se diet) given a moderate-Se diet from weaning; $\cdots \cdots \cdots, \mathrm{B}-2$, 'adapted' rats given a high-Se diet from weaning;

control rats (from dams given a low-Se diet) given a low-Se diet from weaning; $O$, values not significantly different from control values; $\bullet$, values significantly different from control values: $P<0.05 ; \otimes$, controls. 

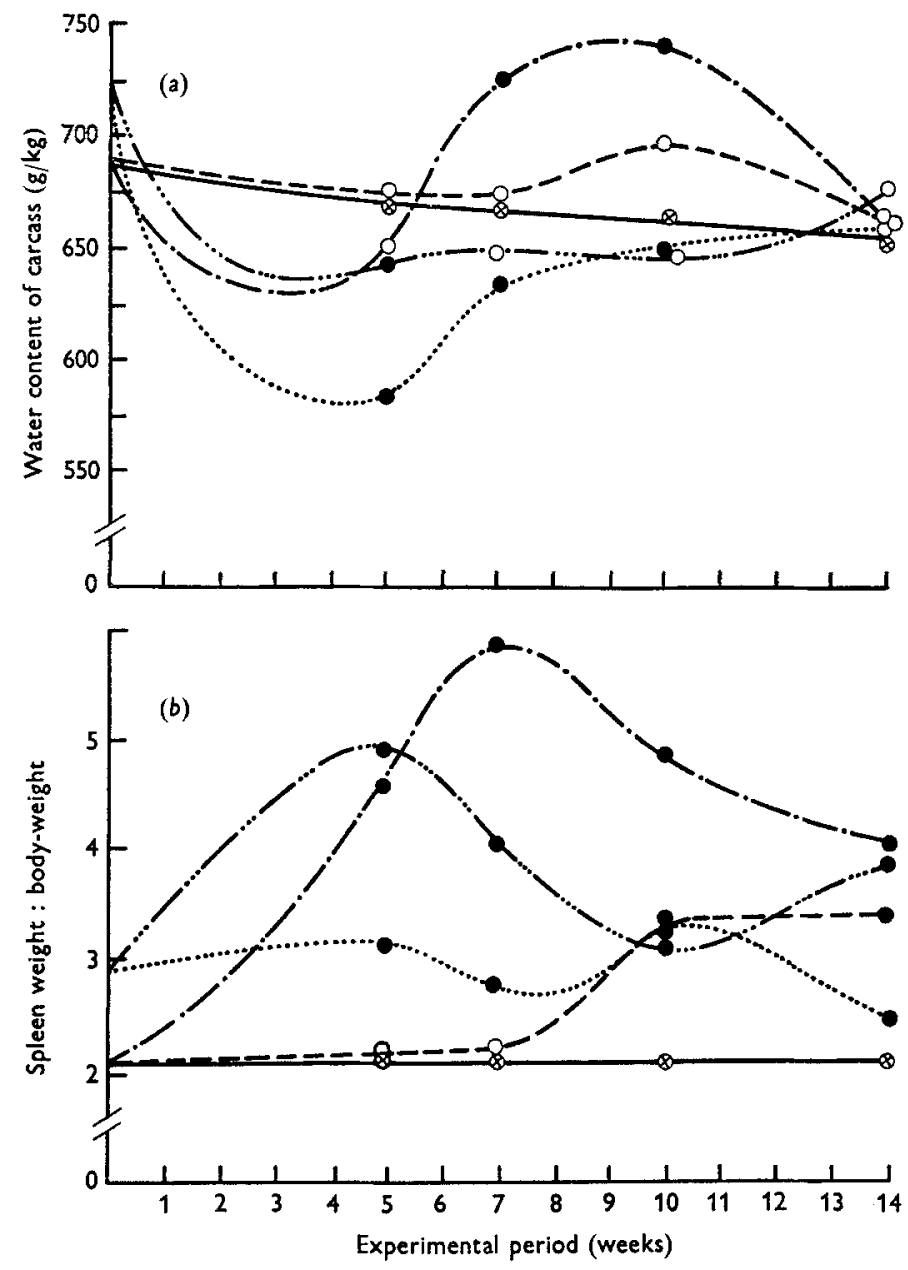

Fig. 3. Effects of dietary selenium on the body composition of rats. (a) Water content of the carcass, $(b)$ ratio, spleen weight $(\mathrm{mg})$ : body-weight $(\mathrm{g}) .----$, A-I, 'non-adapted' rats (from dams given a low-Se diet; $0.5 \mathrm{mg} / \mathrm{kg})$ given a moderate-Se diet $(4.5 \mathrm{mg} / \mathrm{kg})$ from weaning; -.--, A-2, 'non-adapted' rats given a high-Se diet (ro $\mathrm{mg} / \mathrm{kg}$ ) from weaning; ..., B-I, 'adapted' rats (from dams given a moderate-Se diet) given a moderate-Se diet from weaning; ......., B-2 'adapted' rats given a high-Se diet from weaning; -, control rats (from dams given a low-Se diet) given a low-Se diet from weaning; $O$, values not significantly different from control values; $\theta$, values significantly different from control value: $P ₹ 0.05 ; \otimes$, controls.

liver Se content. The rats given the high-Se diet from weaning accumulated Se more slowly and in smaller amounts than the 'non-adapted' rats. Eventually, the rate of elimination of Se exceeded the rate of its accumulation. When given the moderate-Se diet the 'non-adapted' rats (group A) gradually accumulated Se, whereas in the animals of the 'adapted' group B the Se accumulated during gestation was gradually eliminated. Thus, by the end of the $y^{\text {th }}$ week on this diet the Se content of the liver of the 'adapted' rats was significantly lower than that of the 'non-adapted' rats.

Haematological findings. The haemoglobin levels and packed cell volumes (Figs. $2 a$ 

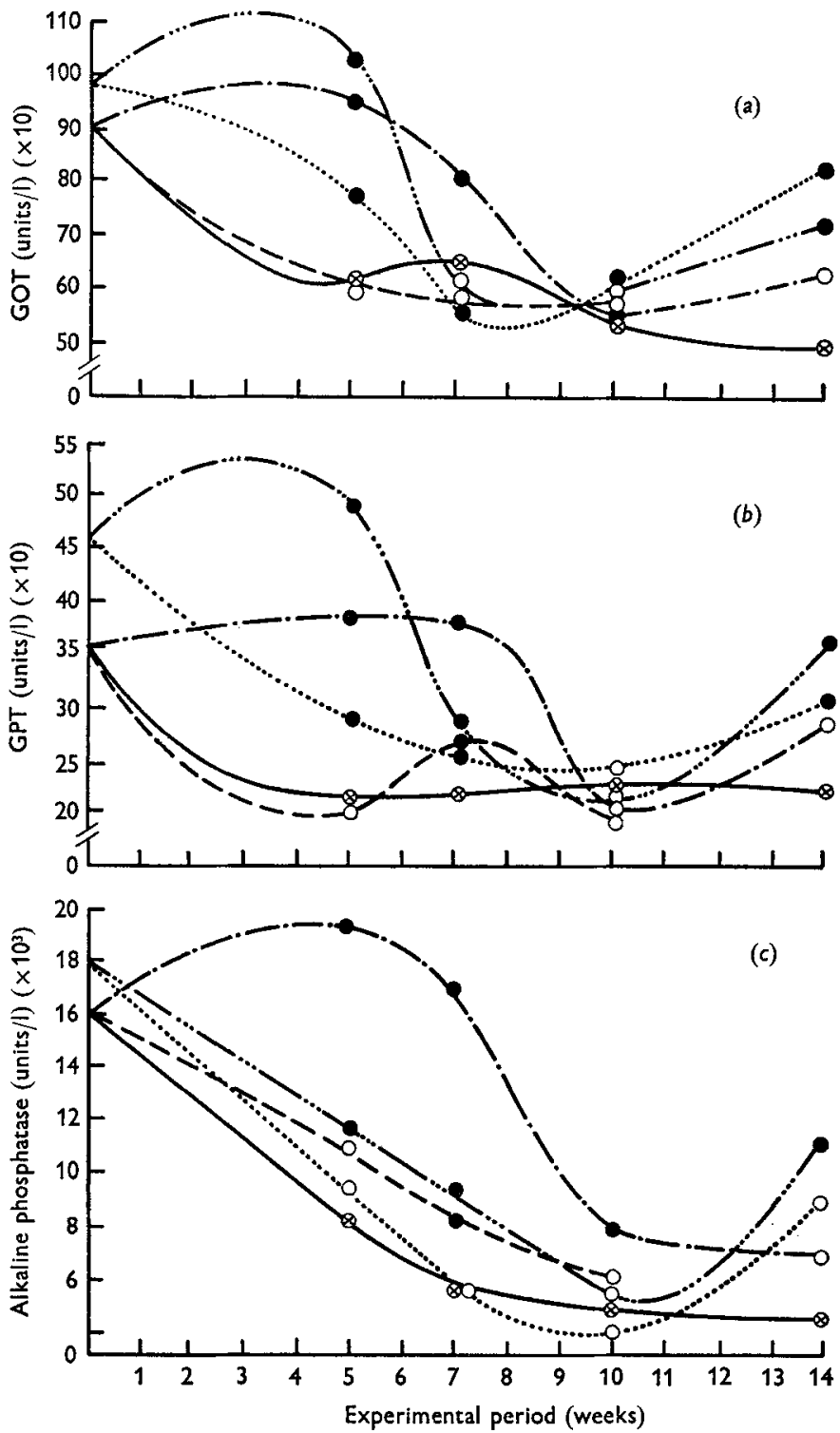

Fig. 4. Effects of dietary selenium on serum enzyme levels. (a) Glutamic-oxaloacetic transaminase (L-aspartate:2-oxoglutarate aminotransferase; $E C$ 2.6.I.I) (GOT), (b) glutamicpyruvic transaminase (L-alanine:2-oxoglutarate aminotransferase; $E C$ 2.6.1.2) (GPT), (c) alkaline phosphatase $(E C$ 3.I.3.I). - - - , A-I, 'non-adapted' rats (from dams given a low-Se diet; $0.5 \mathrm{mg} / \mathrm{kg}$ ) given a moderate-Se diet $(4.5 \mathrm{mg} / \mathrm{kg})$ from weaning; -.-., A-2, 'non-adapted' rats given a high-Se diet (ro $\mathrm{mg} / \mathrm{kg}$ ) from weaning; ...., B-I, 'adapted' rats (from dams given a moderate-Se diet) given a moderate-Se diet from weaning;...$-\cdots$, B-2, 'adapted' rats given a high-Se diet from weaning; _- _, control rats (from dams given a low-Se diet) given a low-Se diet from weaning; $O$, values not significantly different from control values; $\ominus$, values significantly different from control values: $P<0.05$; $\otimes$, controls. For definition of units of activity, see Reitman \& Frankel (I957) (GOT and GPT) and Sigma Chemical Company (1963) (alkaline phosphatase). 
and $b$ ) were lower in the experimental groups than in the respective controls for almost the whole experimental period with the exception of subgroup A-I during the first 7 weeks. The haemoglobin levels and packed cell volumes of the 'adapted' animals at the beginning of the experiment were lower than those of the 'non-adapted' ones and showed a tendency to increase. At the end of the experimental period, the individual values for animals from the same experimental groups showed marked variations. Consequently the differences between the experimental and control groups were not statistically significant except in one instance.

As shown in Fig. $2 c$, prothrombin activities were low by the $5^{\text {th }}$ week and did not change significantly after the 7 th week. Changes in fibrinogen values (Fig. $2 d$ ) were similar to those of prothrombin activity.

Water content and spleen weight. Fig. $3 a$ shows the effects of the various diets on body-water content. Except for subgroup A-I, the body-water content of the experimental animals was lower than that of the controls at the 5 th week, when it started to increase. Only in the 'non-adapted' animals did it reach levels above those of the controls. This was the only instance when the values for the 'adapted' and the 'nonadapted' animals were lower and higher respectively than those of the controls. In each of the groups given the high-Se diet only one instance of ascites was found at the later stages of the experimental period.

Increases in the ratio, spleen weight: total body-weight (i.e. hyperplasia) are shown in Fig. $3 b$. In all instances these increments started early in the experimental period and in subgroup A-2 these values were almost three times greater than control values.

Serum enzymes. The results shown in Fig. 4 indicate that by the 5 th week the activities of serum transaminases in all subgroups except A-r were higher than those of the controls but by the Ioth week they had decreased to control levels. An unexpected increase was noted by the $14^{\text {th }}$ week in subgroups A-2, B-I and B-2. In the instance of alkaline phosphatase activity, these changes were reflected in values at 5 weeks after weaning that were greater than those of the controls. Unfortunately, values for the activities of serum enzymes for subgroup A-I for the $14^{\text {th }}$ week of the experiment were lost.

In Table 2 a summary of the statistical evaluation of the differences between the four experimental groups is shown. The differences between the 'adapted' and the 'non-adapted' rats given the moderate-Se or high-Se diets were more often significant than those between the 'adapted' rats on the moderate-Se and high-Se diets. The only values for which 'adapted' and 'non-adapted' rats did not differ significantly at any stage in the experimental period, were the haemoglobin levels in the animals given the moderate-Se diet and the values for packed cell volume for animals given the high-Se diet.

\section{DISCUSSION}

Effect of adaptation. Values for the rats given the moderate-Se and high-Se diets ( 4.5 and $10 \mathrm{mg} \mathrm{Se} / \mathrm{kg}$ respectively, in the form of Se-containing sesame-seed meal) showed significant differences from control values in all the indices studied. These differences were more pronounced in the rats which were given the high-Se diet. 
Vol. 33

Adaptation to chronic Se intake

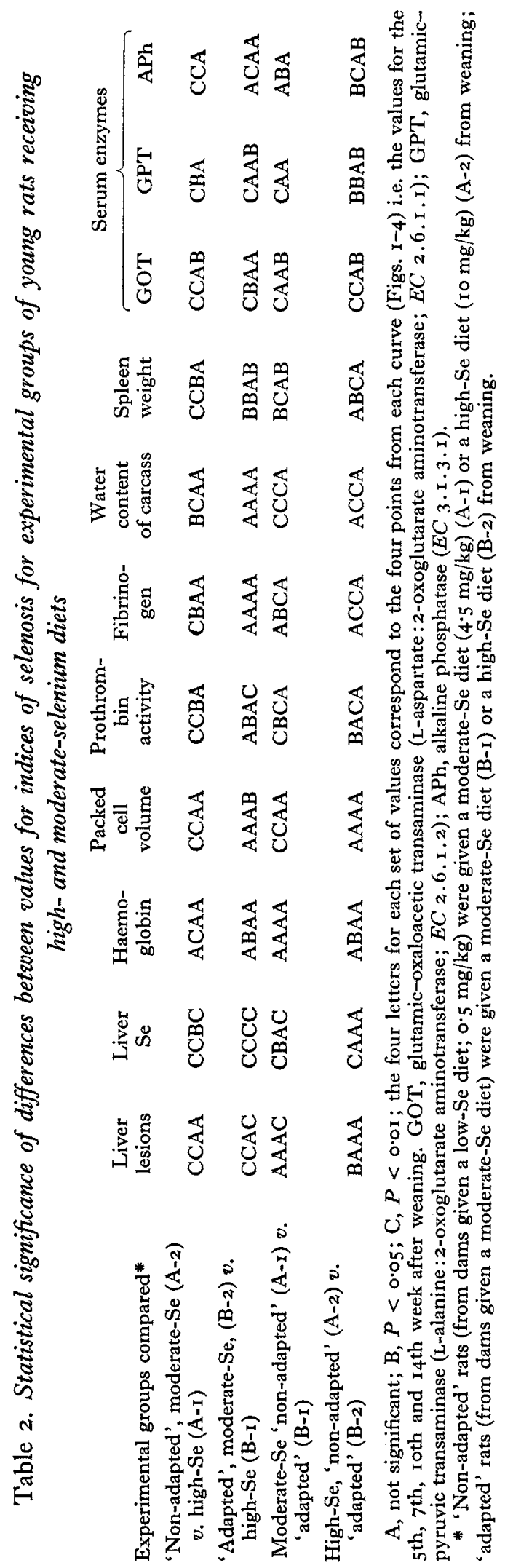


The effects of diets similar in Se content were distinctly more marked in the animals of group A ('non-adapted' rats) than in those of group B ('adapted' rats).

The results of the present experiments, which extended over a period of 14 weeks, confirmed our previous findings, in experiments of shorter duration, regarding adaptation to Se ingestion in the rat (Jaffé \& Mondragón, 1969) and the resulting pathological effects (Jaffé et al. 1972). In the present study, as in our previous studies, liver Se content increased progressively in 'non-adapted' rats in contrast to the Se content of the liver of rats adapted to a moderate-Se diet; in the latter the Se content gradually decreased. It is noteworthy that in the 'adapted' rats the liver Se content at the end of the experiment was significantly lower than that in the 'non-adapted' rats. The rapid increase and the subsequent decrease in liver Se content of animals given the high-Se diet were reported previously (Jaffé \& Mondragón, 1969) although in these earlier studies the experimental period was shorter and therefore the plateau levels, which were lower in the 'adapted' rats at the end of the experimental period than at the beginning (Fig. $\mathrm{I} b$ ), were not found. The number of hepatic lesions and the development of pathological signs in general varied with liver Se levels. None of the signs became worse with continued Se ingestion.

Organic lesions. Beneficial effects can be attributed to what we have termed adaptation, as evidenced by the reduction of hepatic lesions, hyperplasia of the spleen, water accumulation in the carcass, and haematological effects. Moreover, with time there was always a tendency towards smaller deviations from control values.

Body-water content. Halverson, Palmer \& Guss (I966), have reported an increased carcass water content for rats given Se-containing maize. Our studies show that in 'adapted' rats the water increase may occur only after a long experimental period. A comparison between the results shown in Figs. I $a$ and $3 a$ suggests that there is probably no relationship between body-water content and hepatic lesions, since at 5 weeks all the rats which were given the toxic diet had lesions, i.e. at a time when body water was low.

Serum enzymes. The three enzymes studied were selected because in our previous experiments we had observed a significant increase in their levels in the serum of rats after the ingestion of a Se-containing diet (Jaffé et al. 1972). The present results confirm these findings but, at the same time, show that the differences in the enzyme levels between the experimental and control groups disappear after Io weeks (Fig. 4). The significance of the second increase in the enzyme levels after $\mathrm{I}_{4}$ weeks in the rats given Se cannot yet be evaluated because of the limited duration of the experiment.

Individual differences. A significant finding was that in a few animals a severe anaemia developed, along with loss of weight and eventual death, although other rats had a perfectly healthy appearance at the end of the prolonged experimental period. A great difference in individual susceptibility to Se ingestion has also been reported by others (Rosenfeld \& Beath, 1964).

No information on adaptation of farm animals or of humans to chronic Se intake has been reported. It would be of practical importance to know whether effects similar to those described here for rats could be detected in these species. 


\section{REFERENCES}

Cummings, L. M., Martin, J. L. \& Maag, D. D. (x965). Analyt. Chem. 37, 430.

Hainline, A. (1958). Standard Methods of Clinical Chemistry Vol. 2, p. 49. New York: Academic Press. Halverson, A. W., Palmer, I. S. \& Guss, P. L. (I966). Toxic. appl Pharmac. 9, 477.

Jaffé, W. G., Chávez, J. F. \& Mondragón, M. C. (1967). Archos lat.-am. Nutr. 17, 59.

Jaffé, W. G. \& Mondragón, M. C. (1 969). F. Nutr. 97, 431.

Jaffé, W. G., Mondragón, M. C., Layrisse, M. \& Ojeda, A. (1972). Archos lat.-am. Nutr. $22,185$.

King, E. J. \& Delory, G. (1939). Biochem. F. 33, I185.

Natelson, S. (rg6ra). Microtechniques of Clinical Chemistry and ed., p. 213. Springfield, IIl.: C. C. Thomas.

Natelson, S. (196r b). Microtechniques of Clinical Chemistry and ed., p. 74. Springfield, Ill.: C. C. Thomas.

Ohmori, Y. (1937). Enzymologia 4, 217.

Quick, A. J. (I957). Hemorrhagic Diseases. Philadelphia: Lea \& Febiger.

Reitman, S. \& Frankel, S. (1957). Am. F. clin. Path. 28, 56.

Rosenfeld, I. \& Beath, O. A. (1964). Selenium, Geobotany, Biochemistry, Toxicity and Nutrition pp. 14, 178. New York: Academic Press.

Sigma Chemical Company (1963). Sigma Tech. Bull. no. I04.

Smith, M. I. \& Lillie, R. D. (1940). Natn. Inst. Hlth Bull. no. 174.

US Pharmacopeia, XIV (1960). p. 789 . 